\title{
Accessing nanotube bands via crossed electric and magnetic fields
}

\author{
Wade DeGottardi, ${ }^{1}$ Tzu-Chieh Wei, ${ }^{2}$ Victoria Fernández, ${ }^{3,4}$ and Smitha Vishveshwara ${ }^{1}$ \\ ${ }^{1}$ Department of Physics, University of Illinois at Urbana-Champaign, 1110 W. Green Street, Urbana, Illinois 61801-3080, USA \\ ${ }^{2}$ Department of Physics and Astronomy, University of British Columbia, Vancouver, British Columbia, Canada V6T 1Z1 \\ ${ }^{3}$ Instituto de Física La Plata, CONICET 1900 La Plata, Argentina \\ ${ }^{4}$ Departamento de Física, Universidad Nacional de La Plata, CC 67 1900, La Plata, Argentina
}

(Received 19 August 2010; published 7 October 2010)

\begin{abstract}
We investigate the properties of conduction electrons in single-walled armchair carbon nanotubes in the presence of mutually orthogonal electric and magnetic fields transverse to the tube's axis. We find that the fields give rise to an asymmetric dispersion in the right- and left-moving electrons along the tube as well as a band-dependent interaction. We predict that such a nanotube system would exhibit spin-band-charge separation and a band-dependent tunneling density of states. We show that in the quantum dot limit, the fields serve to completely tune the quantum states of electrons added to the nanotube. For each of the predicted effects, we provide examples and estimates that are relevant to experiment.
\end{abstract}

DOI: 10.1103/PhysRevB.82.155411

PACS number(s): 71.10.Pm, 71.20.Tx

\section{INTRODUCTION}

A highlight of graphene-based nanotubes is the presence of a band (valley) quantum degree of freedom which offers a new facet to a range of strongly correlated low-dimensional phenomena. In armchair nanotubes, the bands, which owe their existence to the two-atom basis of the underlying honeycomb lattice, form a pair of gapless linearly dispersing modes. ${ }^{1}$ While a direct consequence of their presence is the measurable quantum conductance of $4 e^{2} / h$, richer effects can arise, for instance, in field-induced orbital moments, ${ }^{2}$ Coulomb-blockade profiles, ${ }^{3}$ and in Kondo physics. ${ }^{4}$ An ability to precisely control the band sector would not only shed light on these effects but also access other band-dependent physics. For instance, in the recently reported nanotube Mott phase, ${ }^{5}$ the nature of this ordered phase could be probed by tuning interband interactions. In the Luttinger liquid-phase typical of interacting one-dimensional systems, spin and charge sectors have been shown to decouple in quantum wires $;{ }^{6}$ nanotubes could extend such fractionalization into yet another sector. Recently, attention has turned toward "valleytronics" as a potential application for quantum information and quantum devices in the parent system of graphene ${ }^{7}$ the ability to manipulate spin and band quantum states in nanotubes would offer an attractive alternative. However, save for some exceptions, ${ }^{2,8}$ the band degree of freedom has thus far remained relatively resilient to any controlled coupling.

In this work, we explore the possibility of coupling to the band sector via applied transverse fields. Electric and magnetic fields individually have been shown to alter the lowenergy properties of conduction electrons. ${ }^{9,10}$ Here, building on our prior work, ${ }^{11}$ we find that the transverse field configuration shown in Fig. 1 in which both fields are present offers a powerful means of accessing the band sector. The fields break time-reversal, particle-hole, and band symmetries, and in fact, render the dispersion asymmetric in the right- and left-moving electrons. Furthermore, the effective Coulomb interaction is modified from its field-free form to include couplings between the charge and band sectors. As a conse- quence, nanotubes in transverse fields could provide a realization of a tunable asymmetric Luttinger liquid. ${ }^{12-14}$ Most pertinently, the system displays spin-band-charge separation, acts as a band selector for electrons tunneling into the nanotube and exhibits a variety of tunable quantum dot shellfilling configurations.

\section{MODEL}

The setup of interest, as shown in Fig. 1, consists of an armchair nanotube [a tube described by a chiral vector of the form $(n, n)$, see Ref. 1 for definition] lying along the $x$-axis subject to an applied magnetic field $\vec{B}=-B \hat{y}$ and electric field $\vec{E}=E \hat{z}$ (see Ref. 15 for a possible experimental implementation). For a tube of diameter $d=3 n a_{c} / \pi$, where $a_{c}$ $\approx 0.142 \mathrm{~nm}$ is the carbon-carbon bond length, the effect of the fields can be characterized by the parameters $b$ $=\sqrt{3} B|e| d^{2} / 4 \hbar$ and $u=|e| E d / 2 t$, where $t \approx 2.7 \mathrm{eV}$ (Ref. 1) is the electron hopping strength between neighboring sites. Near half-filling, the field-free low-energy electronic disper-

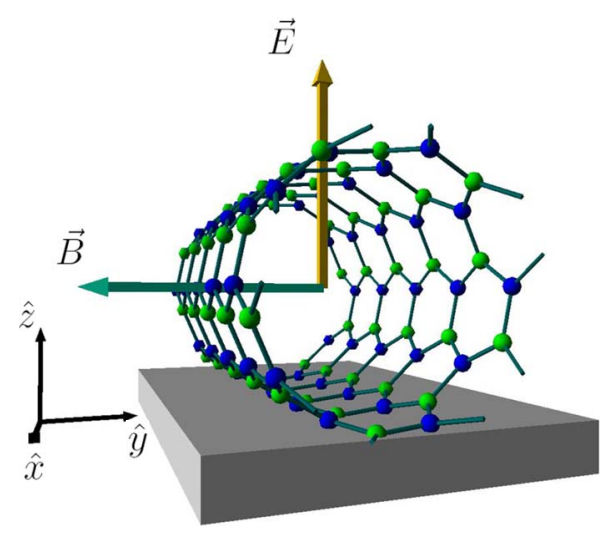

FIG. 1. (Color online) An armchair carbon nanotube in the presence of transverse magnetic (pointing in the $-\hat{y}$ direction) and electric fields (pointing in the $\hat{z}$ direction). The carbon atoms belonging to the $A$ and $B$ sublattices are indicated by dark and light shading, respectively. 
sion, which can be traced to bonding and antibonding states of the underlying graphene sublattices, consists of four degenerate gapless linear modes each having a Fermi velocity $v_{F}=\frac{3 t a_{c}}{2 \hbar} \approx 8 \times 10^{5} \mathrm{~m} / \mathrm{s}$. These modes correspond to right and left movers (indexed by $r= \pm=R / L$ ) at two Fermi points (indexed by $\alpha= \pm$ ) which define the band degrees of freedom.

In this Hall-type setup, classically, each of the fields causes a spiraling motion of electrons, and thus for fixed kinetic energy, reduces their linear velocity along the tube's axis. Moreover, the nonvanishing Poynting vector $\vec{E} \times \vec{B}$ affects right and left movers differently. Indeed, a detailed perturbative band-structure calculation ${ }^{11}$ for large enough tubes $(10 \leqq n<\pi / u)$ shows that the right- and left-moving speeds are given by $v_{r} \approx v_{F}\left(1-\frac{1}{3} b^{2}-\frac{n^{2}}{\pi^{2}} u^{2} \pm \frac{n}{\pi} b u\right)$. The spectrum remains gapless, ${ }^{16}$ but the two Fermi points reside at energies differing by $\Delta_{F} \approx 2 \pi t b u /(\sqrt{3} n)$, indicating a mild banddegeneracy breaking even at the band structure level. The corresponding low-energy noninteracting asymmetric Hamiltonian can be expressed as

$$
H_{0}=-i \hbar \sum_{r \alpha \sigma} \int d x r v_{r} \psi_{r \alpha \sigma}^{\dagger} \partial_{x} \psi_{r \alpha \sigma},
$$

where the band shift and Zeeman term are left implicit. The operator $\psi$ denotes the annihilation of a fermion and $\sigma= \pm$ correspond to electronic spin components. For tubes of diameter $d=6.78 \mathrm{~nm}(n=50)$ and experimentally accessible field strengths $B=6.4 \mathrm{~T}$ and $E=0.02 \mathrm{~V} / \mathrm{nm}$, we find $v_{R}$ $=0.89 v_{F}$ and $v_{L}=0.77 v_{F}$, yielding a pronounced asymmetry in right- and left-moving velocities. For the remainder of this work, we consider tubes of this diameter, corresponding to ultralarge single-walled tubes. ${ }^{17}$

Transverse fields significantly alter not only the lowenergy band structure but also the nature of Coulomb interactions within the tube. The usual forward scattering contribution, $H_{C 1}=\frac{1}{2} \int d x V \rho_{c+}^{2}(x)$, remains and continues to dominate for larger tubes $(n \geqslant 10) .{ }^{18}$ Here $\rho_{c+}(x)=\rho_{R, c+}$ $+\rho_{L, c+}$ with $\rho_{r, c+}=\sum_{\alpha \sigma} \psi_{r \alpha \sigma}^{\dagger} \psi_{r \alpha \sigma}$ is the net charge density and $V \approx \frac{2 e^{2}}{\kappa} \ln \left(\frac{L}{\pi d}\right)$ is the effective one-dimensional interaction, ${ }^{11,19}$ where $e$ is the charge of an electron, $\kappa$ is the dielectric constant, and $L$ is the length of the tube. More interestingly, a second contribution becomes manifest due to the uneven distribution of charge in the circumferential direction induced by the presence of the applied fields. A careful accounting of circumferential modes shows that this additional effective Coulomb term along the tube has the form

$$
H_{C 2}=\frac{\pi \lambda}{4} \int d x\left(\rho_{R, c-}+\rho_{L, c-}\right)\left(\rho_{R, c+}-\rho_{L, c+}\right),
$$

where $\lambda=4 e^{2} b u h / \pi \kappa$ and $h$ is a numerical factor which depends on the details of the geometry of the underlying graphene lattice; for $n \gtrsim 20$ we have $h \approx(1.46 n-4.60) .{ }^{11}$ Here, $\rho_{r, c-}=\Sigma_{\alpha \sigma} \alpha \psi_{r \alpha \sigma}^{\dagger} \psi_{r \alpha \sigma}$ is the difference in charge density between the two bands. The presence of this interaction term has two origins: (i) the magnetic field couples to the crystal momentum, and thus the Fermi points, giving rise to the contribution involving the charge in the $c$ - sector and (ii) the electric field couples to the net charge imbalance in bonding and antibonding states, or alternatively, to the density difference in the right and left movers in the $c+$ sector. This interaction, which is key to the physics studied here, goes a step beyond the usual spin-charge-separating Coulomb term by directly coupling to the band degrees of freedom.

\section{BOSONIZATION}

As with the field-free case, the asymmetric gapless modes and Coulomb interactions can be studied via bosonization, ${ }^{12-14,20}$ which renders the total Hamiltonian for the tube to be quadratic. The one-dimensional fermionic operators can be bosonized as

$$
\psi_{r \alpha \sigma}=\frac{\eta_{r \alpha \sigma}}{\sqrt{2 \pi a_{c}}} \exp \left[i \alpha k_{F} x+i \varphi_{r \alpha \sigma}\right]
$$

where the $\eta_{r \alpha \sigma}$ 's are the so-called Klein factors which enforce anticommutation relations between different channels. ${ }^{19}$ The chiral bosonic fields satisfy the commutation relations $\left[\varphi_{r \alpha \sigma}(x), \varphi_{r^{\prime} \alpha^{\prime} \sigma^{\prime}}\left(x^{\prime}\right)\right]=-i \pi r \delta_{r r^{\prime}} \delta_{\alpha \alpha^{\prime}} \delta_{\sigma \sigma^{\prime}} \operatorname{sgn}\left(x-x^{\prime}\right)$. The density associated with each sector takes the form $\rho_{r \alpha \sigma}$ $=r \partial_{x} \varphi_{r \alpha \sigma} / 2 \pi$. Given the asymmetric dispersion and current dependent interaction, we explicitly employ these chiral fields to ensure that this algebra is preserved. It is convenient to introduce a spin and channel decomposition for the chiral fields, $\varphi_{r, \alpha \sigma}=\frac{1}{2}\left(\varphi_{r, c+}+\alpha \varphi_{r, c_{-}}+\sigma \varphi_{r, s+}+\alpha \sigma \varphi_{r, s-}\right)$. The total kinetic energy takes the bosonized form

$$
H_{0}=\frac{1}{4 \pi} \sum_{a=c / s \pm} \int d x\left[v_{R}\left(\partial_{x} \varphi_{R, a}\right)^{2}+v_{L}\left(\partial_{x} \varphi_{L, a}\right)^{2}\right]
$$

and the total interaction term, $H_{i n t} \equiv H_{C 1}+H_{C 2}$, takes the form

$$
\begin{aligned}
H_{i n t}= & \frac{V}{2 \pi^{2}} \int d x\left(\partial_{x} \varphi_{R, c+}-\partial_{x} \varphi_{L, c+}\right)^{2} \\
& +\frac{\lambda}{4 \pi} \int d x\left(\partial_{x} \varphi_{R, c+}+\partial_{x} \varphi_{L, c+}\right)\left(\partial_{x} \varphi_{R, c-}-\partial_{x} \varphi_{L, c-}\right) .
\end{aligned}
$$

As discussed in previous work on asymmetric bosonization, ${ }^{12-14,20}$ in diagonalizing the full Hamiltonian $H=H_{0}+H_{i n t}$, care needs to be taken to ensure that the diagonal basis preserves the chiral commutation rules respected by the original fields, $\varphi_{r \alpha \sigma}$. Diagonalization requires care due to the unusual commutation relations (the so-called KacMoody algebra) among chiral fields. ${ }^{21}$ Here, we circumvent unwieldy manipulations involving Bogoliubov transformations by employing the trick of converting left-handed fields to right-handed fields via the transformation $\varphi_{L a} \mapsto i \varphi_{L a}$, which then allows for standard rotations.

This method makes it clear that as far as the diagonalization of the Hamiltonian is concerned, the asymmetric dispersion simply shifts the values of the plasmon velocities but has no effect on the eigenvectors. Consider a generic Hamiltonian of the form $H=H_{0}+H_{i n t}$ with $n$ channels $a=1, \ldots, n$ with 


$$
\begin{aligned}
H_{0} & =\frac{1}{4 \pi} \sum_{a} \int d x\left[v_{R}\left(\partial_{x} \varphi_{R, a}\right)^{2}+v_{L}\left(\partial_{x} \varphi_{L, a}\right)^{2}\right] \\
& =\frac{1}{4 \pi} \int d x \vec{\varphi}^{T} \mathcal{H}_{0} \vec{\varphi},
\end{aligned}
$$

where $\quad \vec{\varphi}=\left(\varphi_{R, 1}, \ldots, \varphi_{R, n}, \varphi_{L, 1}, \ldots, \varphi_{L, n}\right)$, $=\operatorname{diag}\left(v_{R}, v_{R}, \ldots, v_{L}, v_{L}, \ldots,\right)$, and $H_{\text {int }}$ is a bilinear of the various densities $\rho_{R / L, a}$. Now, after the transformation $\varphi_{L, a} \mapsto i \varphi_{L, a}$ we have $\mathcal{H}_{0} \mapsto \mathcal{H}_{0}^{\prime}$ with

$$
\begin{aligned}
\mathcal{H}_{0}^{\prime} & =\operatorname{diag}\left(v_{R}, v_{R}, \ldots,-v_{L},-v_{L}\right) \\
& =\operatorname{diag}(v, v, \ldots,-v,-v, \ldots,)+\epsilon I,
\end{aligned}
$$

where $v=\left(v_{R}+v_{L}\right) / 2, \epsilon=\left(v_{R}-v_{L}\right) / 2$, and $I$ is the $2 n \times 2 n$ identity matrix. It is clear from Eq. (7) that $\epsilon$ serves to merely shifts the resulting eigenvalues and that the eigenvectors are independent of $\epsilon$. While this fact limits the role that the asymmetry plays in certain bulk quantities (such as the tunneling density of states), the asymmetry can still play a crucial role in properties such as boundary and impurity scattering and in particular quantum dot physics.

\section{PHYSICAL PROPERTIES}

The coupling between the $c \pm$ sectors [Eq. (2)] plays a central role in the altered physical properties of the tube. The plasmon modes for the coupled charge sectors $c \pm$ move with four different velocities

$$
\begin{gathered}
v_{R / L, 1 / 2}= \pm \epsilon+v_{1 / 2} \\
v_{1 / 2}=\frac{v}{\sqrt{2} K_{c+}}\left[1+K_{c+}^{2} \pm \sqrt{\left(1-K_{c+}^{2}\right)^{2}+\left(\frac{2 K_{c+} \lambda}{\hbar v}\right)^{2}}\right]^{1 / 2}
\end{gathered}
$$

where $v=\left(v_{R}+v_{L}\right) / 2$ is the average velocity of the noninteracting fermions, $\epsilon=\left(v_{R}-v_{L}\right) / 2$ the asymmetry, and $K_{c+}$ $\equiv 1 / \sqrt{1+4 V / \pi \hbar v}$ is the standard Luttinger parameter. The spin sectors $s \pm$ each retain their band structure velocities $v_{R / L}$.

The existence of the two different plasmon velocities $v_{1 / 2}$ demonstrates that transverse fields can access the band sector, which is normally resilient to any bulk coupling. Thus far, momentum resolved bulk tunneling experiments on quantum wires ${ }^{6}$ have revealed two distinct plasmon dispersions corresponding to spin and charge modes; analogous experiments on nanotubes in transverse fields ought to unveil the three pairs of velocities predicted above. For example, for a tube $d=6.78 \mathrm{~nm}$ and experimentally accessible field strengths $B=6.4 \mathrm{~T}$ and $E=0.02 \mathrm{~V} / \mathrm{nm}$, the plasmon velocities are given by $v_{R, 1}=4.07 v_{F}, v_{L, 1}=3.95 v_{F}, v_{R, 2}=0.32 v_{F}$, and $v_{L, 2}=0.20 v_{F}$ for the charge sectors and $v_{R}=0.89 v_{F}$ and $v_{L}=0.77 v_{F}$ for the spin sectors. The observation of three distinct modes would reflect complete splintering of the nanotube electron into its spin, charge, and band sectors. Figures 2 and 3 show the plasmon velocities of a nanotube in the presence of a $12 \mathrm{~T}$ magnetic field as a function of electric field strength.

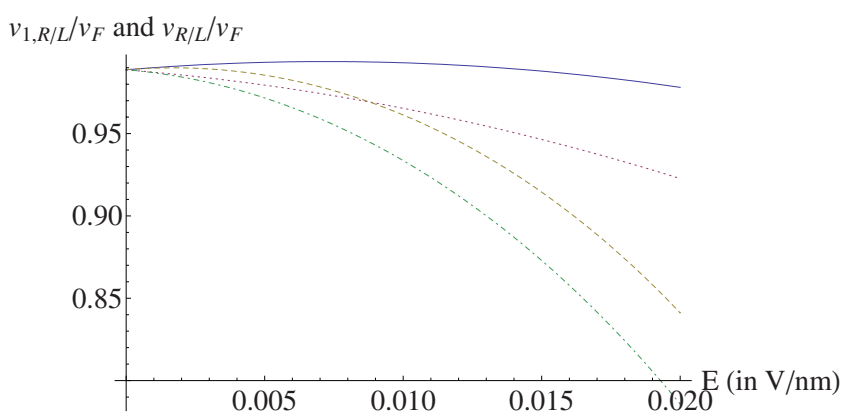

FIG. 2. (Color online) Plasmon velocities $v_{1, R}$ and $v_{1, L}$ and band structure velocities $v_{R}$ and $v_{L}$ (solid, dotted, dashed, and dotteddashed, respectively) for a $1-\mu \mathrm{m}$-long, 4.75-nm-diameter armchair tube in a $12 \mathrm{~T}$ magnetic field as a function of electric field strength.

The effects of transverse fields on the low-energy nanotube physics, particularly in the band degree of freedom, are prominently manifested in physical observables. The tunneling density of states, an ubiquitous, experimentally accessible quantity, retains its power-law form $\chi(E) \sim E^{\beta}$ characteristic of Luttinger liquids but exhibits a modified exponent. Using standard procedures ${ }^{22}$ that now account for fielddependent effects, we find that the tunneling exponent, to lowest order in $\lambda$, is given by

$$
\beta_{r \alpha}=\frac{1}{8}\left(\frac{1}{K_{c+}}+K_{c+}-2\right)-\frac{\lambda}{4 \hbar v} r \alpha\left(\frac{1-K_{c+}}{1+K_{c+}}\right) .
$$

The first part of the exponent, also present in the field-free case, reflects the suppression in tunneling due to interactions (where $K_{c+}$ is now tunable). The second part, which depends on the tunable coupling $\lambda$ of $H_{C 2}$, further suppresses or enhances tunneling depending on the sign of $r \alpha$. As an estimate, for a $d=6.78 \mathrm{~nm}$ tube in a $6.4 \mathrm{~T} B$ field and $0.02 \mathrm{~V} / \mathrm{nm}$ $E$ field with a field-free value of $K_{c+}=0.23$ (corresponding to a $1-\mu \mathrm{m}$-long tube), we have distinctly different exponents $\beta_{R+}=\beta_{L_{-}}=0.22$ and $\beta_{R-}=\beta_{L_{+}}=0.53$. The form of Eq. (9) reflects band (valley) selection; for example, a right-moving electron would preferentially tunnel into the $\alpha=+$ Fermi point for the field configuration shown in Fig. 1.

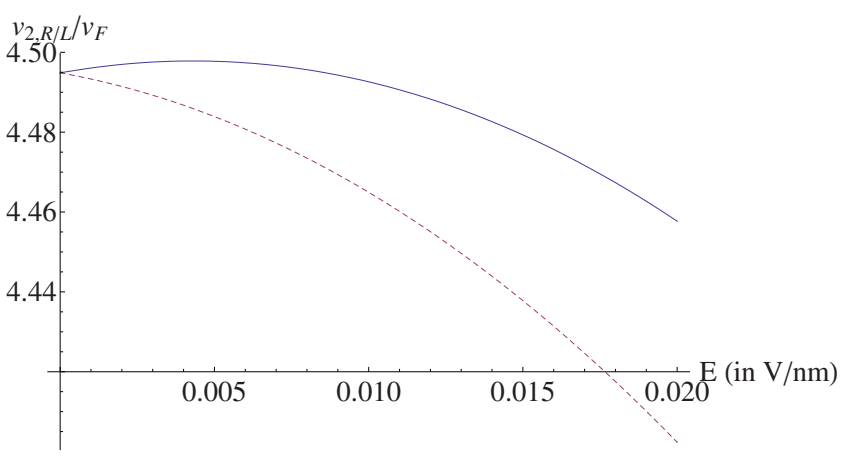

FIG. 3. (Color online) Plasmon velocities $v_{2, R}$ and $v_{2, L}$ (solid and dashed, respectively) for a $1-\mu \mathrm{m}$-long, 4.75-nm-diameter armchair tube in a $12 \mathrm{~T}$ magnetic field as a function of electric field strength. The field-free charge plasmon velocity of this tube would be $v_{\rho}=4.52 v_{F}$. 
A physical consequence of transverse fields yielding such a band-dependent exponents would be the presence of two different power-law contributions to the non-Ohmic conductance of the nanotube. ${ }^{23}$ On a related note, the presence of an impurity would distinguish these exponents; conductance properties would be sensitive to whether or not an electron impinging on the impurity switched band index. Banddependent effects similar to those in the density-of-states exponent would be manifest in any susceptibilities involving the sublattice degree of freedom. Tendencies toward different orderings, such as charge or spin density waves, which are governed by associated susceptibilities, would in turn reflect band dependence. For instance, charge density waves have contributions from operators $\hat{O}_{C D W \pi}^{ \pm} \sim \sum_{r \alpha \sigma} r \psi_{r \alpha \sigma}^{\dagger} \psi_{-r \pm \alpha \sigma}$, reflecting like (+) and staggered (-) band correlations at $A$ and $B$ sublattice sites. While these operators both come on an equal footing in the field-free case, we find that fields discriminate between these two ordering tendencies. In particular, to first order in $(\lambda / v), O_{C D W \pi}^{+}$is unaffected by the presence of fields, whereas $O_{C D W \pi}^{-}$'s scaling dimension becomes

$$
\eta_{-}=\frac{3+K_{c+}^{0}}{4}-\frac{2 K_{c+}^{0}}{1+K_{c+}^{0}} \frac{|\lambda|}{v},
$$

where $K_{c+}^{0}$ is the field-free value of the Luttinger parameter. Thus, the operator $O_{C D W \pi}^{-}$is rendered more relevant. Fields can thus differentiate between two types of band-dependent ordering at the sublattice level.

\section{QUANTUM-DOT PHYSICS}

In the quantum-dot limit achieved by high resistance contacts or sufficiently low temperatures, we find that transverse fields enable controlled tuning of Coulomb blockade physics and nanotube quantum states. Here, as in previous treatments, ${ }^{18,24,25}$ we describe the dot as a finite-sized version of the net nanotube Hamiltonian and focus on the topological sectors as relevant for standard quantum dot experiments involving adiabatic tuning. Field-induced interactions give rise to a Coulomb blockade structure in which the charge and band degrees of freedom are coupled. The coupling between the $c+$ and $c-$ sectors arises due to the interaction $H_{C 2}$ [Eq. (2)] and the unusual form of the current operators. In particular, the charge current differs from the naïve form $J_{c+}$ $=v_{R} \rho_{R, c_{+}}-v_{L} \rho_{L, c+}$ due to $H_{C 2}$. The form of the current is easily derived by noting that the current $J_{a}$ for every sector obeys a continuity equation $\partial_{t} \rho_{a}=-\partial_{x} J_{a}$. For the $c+$ sector, we obtain

$$
\begin{aligned}
\partial_{t} \rho_{c+} & =i\left[H, \rho_{R, c+}+\rho_{L, c+}\right] \\
& =-\partial_{x}\left(v_{R} \rho_{R, c+}-v_{R} \rho_{L, c-}+\lambda \rho_{c-}\right)
\end{aligned}
$$

thus giving $J_{c+}=v_{R} \rho_{R, c_{+}}-v_{R} \rho_{L, c_{-}}+\lambda \rho_{c-}$. For $a=c-, s \pm$ we have $J_{a}=v_{R} \rho_{R, a}-v_{L} \rho_{L, a}$.

The Coulomb blockade Hamiltonian follows from requiring $\rho_{\alpha \sigma} \equiv \Sigma_{r} \rho_{r, \alpha \sigma}=N_{\alpha \sigma} / L$, where the $N_{\alpha \sigma}$ 's are integers and the no-current condition $J_{a}=0$. The channel occupation numbers are defined $N_{c \pm}=\left(N_{+\uparrow}+N_{+\downarrow}\right) \pm\left(N_{-\uparrow}+N_{-\downarrow}\right)$ and $N_{s \pm}$ $=\left(N_{+\uparrow}-N_{+\downarrow}\right) \pm\left(N_{-\uparrow}-N_{-\downarrow}\right)$. In the presence of a gate voltage

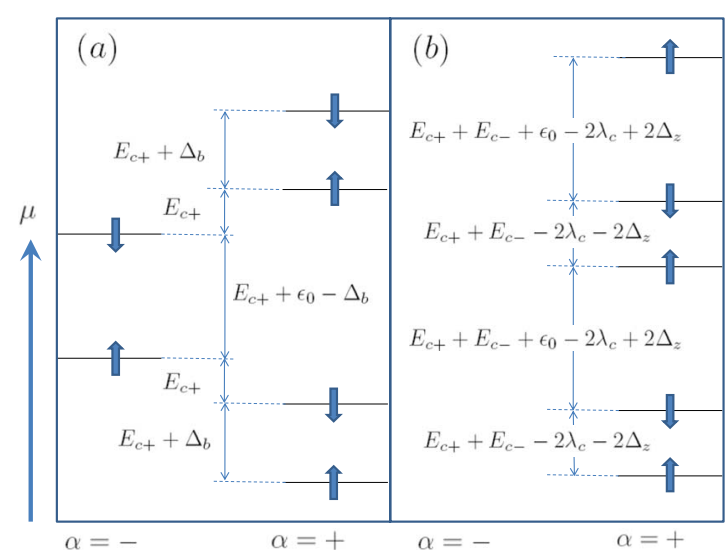

FIG. 4. (Color online) Examples of shell filling. Figure indicates the order, $\alpha$ point, and spin of tunneled electron with increasing chemical potential $\mu$ (not drawn to scale). (a) Filling order for a tube in the absence of fields and with $\Delta_{b} \neq 0$. (b) Shell filling for $N_{c+}=N_{c-}$ exhibits twofold periodicity and complete polarization into band $\alpha=+$. This condition is approximately met for a $1 \mu \mathrm{m}$ tube of diameter $6.78 \mathrm{~nm}$ in the presence of magnetic and electric fields $4.93 \mathrm{~T}$ and $0.0242 \mathrm{~V} / \mathrm{nm}$, respectively (see text for specific values of the Coulomb blockade parameters for this case and other details).

$V_{G}$, the resulting Hamiltonian characterized by quantum numbers $N_{a}$ for each topological channel is given by

$$
\begin{aligned}
H_{T}= & \sum_{a=c / s \pm} \frac{\epsilon_{a}}{8} N_{a}^{2}-\mu N_{c+}-\frac{\pi \lambda}{4 L}\left(\frac{v_{R}-v_{L}}{v_{R}+v_{L}}\right) N_{c+} N_{c-}+\frac{1}{2} \Delta_{b} N_{c-} \\
& -\Delta_{Z} N_{s+},
\end{aligned}
$$

where $\epsilon_{a}=\epsilon_{0}+4 E_{a}, \epsilon_{0}=\frac{2 \pi \hbar v_{R} v_{L}}{L\left(v_{R}+v_{L}\right)}$, and $E_{a}$ is the interaction strength in a given mode. Here, $E_{c+}=V / L, E_{c-}$ $=-\pi \lambda^{2} / 4 \hbar v L$, and $E_{s \pm}=0$. The term $\mu$ is proportional to $e V_{G}$, and $\Delta_{Z}=\mu_{B} B$ accounts for the Zeeman splitting. The band splitting $\Delta_{b}$ will depend on boundary conditions; in the absence of Fermi point mixing at the tube ends, it reduces to the band structure Fermi point mismatch $\Delta_{F} \cdot{ }^{11}$ As typical parameter values, for a $1 \mu \mathrm{m}$ tube of diameter $6.78 \mathrm{~nm}$, and magnetic and electric field strengths of $4.93 \mathrm{~T}$ and 0.0242 $\mathrm{V} / \mathrm{nm}$, we find $\epsilon_{0}=1.36 \mathrm{meV}, E_{c+} / \epsilon_{0}=5.93, E_{c-} / \epsilon_{0}=-0.23$, $\Delta_{Z} / \epsilon_{0}=0.21$, and $\lambda / L \epsilon_{0}=0.24$.

By virtue of their field dependence, several parameters in $H_{T}$ can be varied to access a wide variety of shell filling configurations of the nanotube quantum dot in Coulomb blockade experiments. The configurations correspond to sets of electron occupation numbers which minimize the energy associated with Eq. (12). ${ }^{11,18}$ While the associated parameter space is too extensive for an exhaustive study, a few salient characteristics of shell filling are as follows. (i) In actual experiments, ${ }^{3,26}$ the band degeneracy is often naturally broken due to physical attributes such as the confining potential created by the leads, yielding patterns such as in Fig. 4(a). Here, the field dependence of $\Delta_{b}$ enables controlled tuning of band degeneracy breaking as well as probing the extent of Fermi point mixing at the tube ends. ${ }^{11,27}$ (ii) A fourfold periodicity has been observed in some experiments reflecting 
the band and spin degeneracy of the tube. ${ }^{3}$ (iii) As a direct demonstration of band tuning, the parameter values quoted above yield the twofold periodic shell-filling pattern in Fig. 4(b). In particular, these values are chosen such that Eq. (12) is minimized by the condition $N_{c+}=N_{c-}$, entirely restricting the tunneling into a given $\alpha$ point. We remark that this condition requires a fine tuning of fields, and also that other field values can even render $\epsilon_{c-}$ negative, resulting in an instability toward complete polarization into one band. (iv) Periodicity can also be entirely obliterated by choosing an irrational ratio between two of the relevant shell-filling parameters. As demonstrated above, transverse fields provide a precise means of preparing and manipulating the electronic spin and band quantum numbers of the nanotube quantum dot.

\section{CONCLUSION}

We have presented transverse fields as powerful probes to access and explore a rich range of physics in armchair nano- tubes that directly addresses the band degree of freedom. We have found that these fields induce an unusual interaction that couples the charge and band sectors. We have predicted that signatures of these field effects will be apparent in a variety of measurements including those probing Luttinger liquid parameters and plasmon structure, tunneling density of states and impurity scattering. We have shown that transverse fields can be used for controlled manipulation of nanotube quantum-dot states, making this proposed setup a potential building block for nanoscale quantum devices.

\section{ACKNOWLEDGMENTS}

We would like to thank N. Mason and M. Stone for their perceptive comments. This work is supported by NSF under Grant No. DMR09-06521 (W.D. and S.V.), NSERC and MITACS (T.-C.W.), and UNLP and CONICET (V.F.)
${ }^{1}$ R. Saito, G. Dresselhaus, and M. S. Dresselhaus, Phys. Rev. B 50, 14698 (1994).

${ }^{2}$ F. Kuemmeth, S. Ilani, D. C. Ralph, and P. L. McEuen, Nature (London) 452, 448 (2008).

${ }^{3}$ W. Liang, M. Bockrath, and H. Park, Phys. Rev. Lett. 88, 126801 (2002).

${ }^{4}$ T. W. Odom, J.-L. Huang, C. L. Cheung, and C. M. Lieber, Science 290, 1549 (2000).

${ }^{5}$ V. V. Deshpande, B. Chandra, R. Caldwell, D. S. Novikov, J. Hone, and M. Bockrath, Science 323, 106 (2009).

${ }^{6}$ O. M. Auslaender, H. Steinberg, A. Yacoby, Y. Tserkovnyak, B. I. Halperin, K. W. Baldwin, L. N. Pfeiffer, and K. W. West, Science 308, 88 (2005).

${ }^{7}$ A. Rycerz, J. Tworzydlo, and C. W. J. Beenakker, Nat. Phys. 3, 172 (2007).

${ }^{8}$ D. V. Bulaev, B. Trauzettel, and D. Loss, Phys. Rev. B 77, 235301 (2008).

${ }^{9}$ S. Bellucci and P. Onorato, Eur. Phys. J. B 47, 385 (2005); Ann. Phys. 321, 934 (2006); Eur. Phys. J. B 52, 469 (2006).

${ }^{10}$ D. S. Novikov and L. S. Levitov, Phys. Rev. Lett. 96, 036402 (2006); H.-W. Lee and D. S. Novikov, Phys. Rev. B 68, 155402 (2003).

${ }^{11}$ W. DeGottardi, T.-C. Wei, and S. Vishveshwara, Phys. Rev. B 79, 205421 (2009).

${ }^{12}$ M. Trushin and A. L. Chudnovskiy, EPL 82, 17008 (2008).

${ }^{13}$ V. I. Fernández, A. Iucci, and C. M. Naon, Eur. Phys. J. B 30, 53 (2002).

${ }^{14}$ G. Refael, J. Heo, and M. Bockrath, Phys. Rev. Lett. 98, 246803
(2007).

${ }^{15}$ D. Padmaraj, W. Zagozdzon-Wosik, L.-M. Xie, V. G. Hadjiev, P. Cherukuri, and J. Wosik, Nanotechnology 20, 035201 (2009).

${ }^{16}$ For nonperpendicular fields oriented at an angle $\chi$, a relevant coupling between right- and left-moving electrons opens a bare gap of order $t b u\left(2 \sqrt{3} \cos \frac{\pi}{3 n}\right)|\cos \chi| /\left(1+2 \cos \frac{\pi}{3 n}\right)$. This gap however is rendered irrelevant by interaction effects.

${ }^{17}$ C. L. Cheung, A. Kurtz, H. Park, and C. M. Lieber, J. Phys. Chem. B 106, 2429 (2002).

${ }^{18}$ C. Kane, L. Balents, and M. P. A. Fisher, Phys. Rev. Lett. 79, 5086 (1997).

${ }^{19}$ R. Egger and A. O. Gogolin, Eur. Phys. J. B 3, 281 (1998).

${ }^{20}$ V. I. Fernandez, Ph.D. thesis, Universidad Nacional de La Plata, 2002.

${ }^{21}$ A. Atland and B. Simons, Condensed Matter Field Theory (Cambridge University Press, Cambridge, 2006).

${ }^{22}$ T. Giamarchi, Quantum Physics in One Dimension (Oxford University Press, Oxford, 2004).

${ }^{23}$ H. W. C. Postma, M. de Jonge, Z. Yao, and C. Dekker, Phys. Rev. B 62, R10653 (2000).

${ }^{24}$ M. Fabrizio and A. O. Gogolin, Phys. Rev. B 51, 17827 (1995).

${ }^{25}$ S. Eggert, H. Johannesson, and A. Mattsson, Phys. Rev. Lett. 76, 1505 (1996).

${ }^{26}$ A. Makarovski, L. An, J. Liu, and G. Finkelstein, Phys. Rev. B 74, 155431 (2006).

${ }^{27}$ E. McCann and V. Fal'ko, J. Phys.: Condens. Matter 16, 2371 (2004). 